REGARDS

SUR L'ECONOMIE ALLEMAND

BULLETIN ECONOMIQUE DU CIRAC
Regards sur l'économie allemande

Bulletin économique du CIRAC

106 | 2012

Varia

\title{
L'arrêt de Karlsruhe à propos du MES
}

\section{Peter-Christian Müller-Graff}

Traducteur : Isabelle Bourgeois

\section{OpenEdition}

\section{Journals}

Édition électronique

URL : http://journals.openedition.org/rea/4464

DOI : $10.4000 /$ rea.4464

ISBN : 978-2-8218-1422-6

ISSN : 1965-0787

Éditeur

CIRAC

Édition imprimée

Date de publication : 27 octobre 2012

Pagination : 5-15

ISSN : 1156-8992

\section{Référence électronique}

Peter-Christian Müller-Graff, «L'arrêt de Karlsruhe à propos du MES », Regards sur l'économie allemande [En ligne], 106 | octobre 2012, mis en ligne le 01 octobre 2014, consulté le 01 mai 2019. URL : http:// journals.openedition.org/rea/4464 ; DOI : 10.4000/rea.4464 


\section{L'arrêt de Karlsruhe à propos du MES}

\section{Peter-Christian Müller-Graff}

\begin{abstract}
L'arrêt rendu le 12 septembre 2012 par le Tribunal constitutionnel fédéral à propos du Mécanisme européen de stabilité (MES) était attendu avec angoisse et fébrilité par l'opinion internationale, surtout celle des Etats de la zone Euro les plus touchés par la crise de la dette. La Cour, qui avait été saisie d'un recours en référé en juillet, n'avait certes pas encore à statuer sur le fond, mais elle n'en devait pas moins se prononcer notamment sur plusieurs questions lourdes de conséquences quant à l'avenir des mécanismes de sauvegarde décidés au sein de la zone Euro.
\end{abstract}

Les juges de Karlsruhe allaient-ils considérer que les lois d'approbation allemandes du Traité instituant le MES avaient été adoptées en violation de la responsabilité budgétaire du Bundestag inscrite dans la Loi fondamentale et accorder protection juridique aux plaignants, ce qui serait revenu à interdire au moins durant quelque temps la participation de l'Allemagne au MES et à priver de ce soutien budgétaire les Etats en difficulté, notamment l'Italie, l'Espagne, le Portugal, la Grèce et Chypre? On le redoutait. L'imprécision dans la formulation de certains articles du Traité instituant le MES résisterait-elle à l'exigence constitutionnelle de prévisibilité des charges budgétaires issues d'engagements internationaux ? On en doutait. La Cour fixerait-elle un plafond chiffré aux aides que la Constitution autorise l'Allemagne à verser dans le contexte de l'UEM ? On s'inquiétait...

Il est vrai que jamais, dans l'histoire de la Cour, un recours en constitutionnalité n'avait rassemblé autant de plaignants de différentes obédiences. Cette large participation révélait aussi que le recours en constitutionnalité jouait comme une soupape de sécurité autorisant l'expression d'une profonde insatisfaction avec la politique transnationale d'aides budgétaires menée au sein de la zone Euro depuis mai 2010. Le soulagement fut donc grand - en Allemagne comme dans les autres Etats de l'UE - quand Karlsruhe prononça un « oui, mais "...

En réalité, il était n'était guère justifié de redouter que la Cour allait enrayer le processus de ratification du Traité sur le MES en émettant des réserves de principe d'ordre constitutionnel contre la politique d'aide budgétaire transnationale. Car une analyse sereine des questions qui lui étaient soumises, à la lumière d'une part des principes directeurs qu'il avait énoncés dans son arrêt du 7 septembre 2011 sur les aides budgétaires dans la zone Euro (2 BvR 987/10, 2 BvR 1485/10, 2 BvR 1099/10 = BVerfGE 129, 124 ; voir REA 102/2011), et d'autre part de la continuité sans faille de la jurisprudence constructive de Karlsruhe en matière d'intégration européenne, auraient montré que ces craintes étaient dépourvues de tout fondement.

Dans ses attendus, l'arrêt à propos du MES (BVerfG, 2 BvR 13/90/12) concrétise les critères relatifs à l'exigence constitutionnelle de la responsabilité budgétaire du Bundestag (I), analyse la conformité à la Constitution allemande du Traité instituant le MES (II), fait obligation aux organes politiques allemands de prendre des garanties en droit international contre tout engagement de l'Allemagne découlant d'une interprétation divergente du traité (III) et reporte au jugement de fond qu'il rendra ultérieurement la question de la conformité au

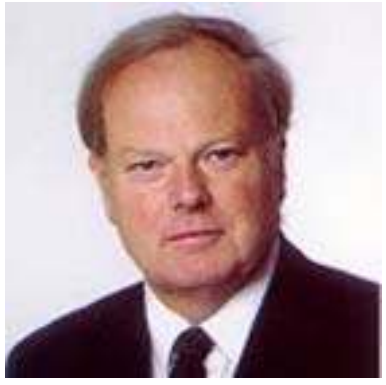

Prof. P.-C. Müller-Graff, Directeur de l'Institut für deutsches und europäisches Gesellschafts- und Wirtschaftsrecht, Université de Heidelberg 
droit de la politique d'aide budgétaire adoptée par la BCE le 6 septembre 2012 (IV).

\section{I - La garantie constitutionnelle de la responsabilité budgétaire du Bundestag}

La responsabilité budgétaire du Bundestag est la pierre angulaire du contrôle en constitutionnalité qu'est cet arrêt à propos du MES. Les considérants sur ses fondements et ses critères d'application s'inscrivent dans la continuité de la jurisprudence du Tribunal constitutionnel fédéral, confèrent à certains de ces critères une portée concrète et justifient l'irrecevabilité d'un certain nombre des recours en constitutionnalité déposés.

\section{Continuité des fondements et critères d'application}

L'autonomie budgétaire du Bundestag est un élément central du processus démocratique

Interdiction du transfert à l'UE de la compétence de la compétence
L'arrêt confirme une fois de plus que le droit de vote inscrit à l'art. 38 de la Loi fondamentale (élection des députés au Bundestag) légitime une saisine individuelle du Tribunal constitutionnel fédéral contre des lois d'approbation de traités internationaux. En effet, le droit de vote garantit que l'auto-détermination des citoyens ainsi que leur participation en toute liberté et égalité au pouvoir exercé par l'Etat dans la République fédérale ne peuvent en aucun cas être vidés de leur substance (al. 209, dans le prolongement notamment des arrêts BVerfGE 89, p. 155 et BVerfGE 123, pp. 267 et 340). Cette auto-détermination et cette participation incluent l'autonomie budgétaire du Bundestag (al. 210, dans le prolongement des arrêts BVerfGE 123, pp. 267 et 359 ; BVerfGE 129, pp. 124 et 177) en tant qu'un élément central du processus démocratique de formation de la volonté du peuple (al. 210, dans le prolongement des arrêts BVerfGE 70, pp. 324 et 355 sq. ; BVerfGE 79, pp. 311 et 329 ; 129, pp. 124 et 177).

Dans son arrêt rendu en 2011 à propos des aides budgétaires dans la zone Euro, le Tribunal constitutionnel fédéral avait rappelé en ces termes le lien entre droit de vote et autonomie budgétaire du parlement: « le droit du citoyen à la démocratie... se trouverait invalidé si le parlement abandonnait des éléments essentiels de l'autodétermination politique et retirait ainsi durablement au peuple son pouvoir d'influence démocratique» (voir REA 102/2011). Or ce principe démocratique se trouve également garanti dans un "système de gouvernance inter-gouvernementale » (al. 211).

L'arrêt de 2012 consolide pareillement les interdictions qui en découlent. A savoir d'une part celle du transfert de la compétence de la compétence à des instances internationales (al. 209, dans le prolongement de BVerfGE 89, pp. 155, 187 sq., 192 et 199 ; BVerfGE 104, pp. 151 et 219 ; BVerfGE 123, pp. 267 et 349) et sa conséquence logique qu'est le principe d'attribution qui veut que l'Union ne dispose que des compétences que lui attribuent les traités.

Le concept de la compétence de la compétence est inhérent au fédéralisme. Il désigne le pouvoir (souveraineté) d'attribuer certaines compétences à un détenteur du pouvoir souverain. Dans le cas de l'Allemagne, la compétence de la compétence revient par principe à la Fédération. C'est elle qui confère les compétences sectorielles à la Fédération ou aux Länder. Comme cette répartition est inscrite dans la Loi fondamentale (art. 73 sq. en ce qui concerne celle des compétences législatives), sa modification ne peut se faire que via une modification de la Constitution (art. $79, \S 1$ et 2 de la Loi fondamentale) qui exige la majorité des deux-tiers du Bundestag et du Bundesrat.

En ce qui concerne l'UE, celle-ci ne dispose pas de la compétence de la compétence, c'est-à-dire qu'elle ne peut pas donner pouvoir. Elle dispose des seuls pouvoirs et compétences que lui ont attribué/transféré par voie de traité les Etats membres : c'est le principe d'attribution (art. 5, § 2 du Traité de l'UE).

L'arrêt conforte d'autre part l'interdiction faite aux organes constitutionnels allemands d'accorder blanc-seing à d'autres acteurs dans l'exercice de la «puissance » publique (al. 209, dans le prolongement de BVerfGE 58, pp. 1 et 37 ; BVerfGE 89, pp. 155, 183 sq. et 187 ; BVerfGE 123, pp. 267 et 351). Et il consolide par ailleurs un certain nombre de critères constitutionnels abstraits relatifs au traitement de mandats nécessitant une interprétation (voir infra). 
S'inscrivent également dans la continuité de la jurisprudence de Karlsruhe les conséquences de l'arrêt du 12 septembre 2012 sur la garantie constitutionnelle de la responsabilité budgétaire du Bundestag. A savoir d'une part l'interdiction qui lui est faite de transférer cette responsabilité à d'autres acteurs en leur conférant des mandats de politique budgétaire indéterminés ou de s'exposer à des mécanismes "susceptibles d'entraîner, sans son approbation constitutive préalable, des charges pour le budget, qu'il s'agisse de dépenses ou d'un déficit de recettes » (al. 212 dans le prolongement de BVerfGE 129, p. 124). D'autre part l'interdiction de voter " tout automatisme en matière de cautions ou de versements non assorti de strictes conditions et non limité dans ses effets » qui, "une fois déclenché ", échapperait au contrôle et à l'influence du Bundestag (al. 213, dans le prolongement de BVerfGE 129, pp. 124 et 180). Enfin, l'interdiction de prendre part à "des mécanismes durables adoptés en droit international... qui auraient pour conséquence la prise en charge de la responsabilité décisionnelle d'autres Etats ».

Ces interdits se doublent d'une exigence: "toute mesure de solidarité d'ampleur importante entraînant des dépenses et prise par la Fédération au niveau international ou au sein de l'Union ... (doit) être approuvée dans le détail par le Bundestag »; et, dans le cas d'accords ayant un « impact structurel » sur le pouvoir budgétaire, doit en outre être "préservée une influence parlementaire suffisante sur la manière dont sont utilisés les moyens mis à disposition" (al. 214 dans le prolongement de BVerfGE 129, pp. 124 et 180sq.). Enfin, l'arrêt en tire une nouvelle conséquence en ce qui concerne le droit à l'information du parlement allemand (voir infra).

Dans leur arrêt à propos du MES, les juges de Karlsruhe réitèrent également leur réticence à déduire du principe de démocratie une limitation contraignante pour l'engagement de dépenses ou la prise de garanties. Et ils rappellent à juste titre l'ampleur du pouvoir budgétaire du législateur, soulignant encore une fois que le Tribunal constitutionnel fédéral se doit de le respecter (al. 216 et 217, dans le prolongement de BVerfGE 129, pp. 124 et 182sq.).

\section{Portée concrète de la garantie de l'auto-détermination en matière de politique budgétaire}

L'arrêt à propos du MES apporte également deux nouveaux critères précisant et concrétisant la garantie d'auto-détermination budgétaire au sens de l'intangibilité de la Constitution (Ewigkeitsklausel, art. 79 , § 3 de la Loi fondamentale) que seule pourrait lever l'adoption d'une nouvelle Constitution.

Le premier critère réside dans la souplesse qu'offre ce principe abstrait pour garantir une interprétation conforme à la Loi fondamentale d'engagements pris dans le cadre de traités internationaux. L'arrêt ne barre nullement - quant au principe - la voie (diplomatique) à la conclusion de tels engagements ; mais il contraint le législateur allemand à prendre "des garanties appropriées lui permettant d'assumer effectivement "(al. 209) la responsabilité en matière d'intégration européenne qu'exige de lui la Loi fondamentale. II exige en conséquence que soient prises des mesures appropriées et suffisantes pour que les engagements internationaux de la République fédérale respectent le cadre imposé par la Loi fondamentale et ses limites.

Le deuxième critère concerne le droit d'information du Bundestag, condition sine qua non pour lui permettre d'assumer sa responsabilité budgétaire globale. L'arrêt étend ce droit, considérant qu'il s'applique aussi aux " affaires de l'Union européenne " tout en l'ancrant expressément dans le «principe d'intangibilité " de la Loi fondamentale (al. 215 en liaison avec sa jurisprudence relative à ce droit d'information : BVerfGE 67, pp. 100 et 130 ; BVerfGE 110, pp. 119 et 225 ; BVerfGE 124, pp. 78 et 114). Ce faisant, l'arrêt confère plus de poids encore à la garantie d'intangibilité de la Constitution. Et il souligne à juste titre que la responsabilité budgétaire globale du Bundestag se trouve également garantie
Interdiction faite au Bundestag de transférer sa responsabilité budgétaire sans strictes conditions

La Cour de Karlsruhe doit respecter le pouvoir budgétaire du législateur

Le Bundestag doit prendre des garanties pour pouvoir assumer effectivement l'intégration européenne de l'Allemagne

Le droit d'information du Bundestag en est une condition sine qua non 
par le droit primaire de l'UE (Traité de l'UE et Traité sur le fonctionnement de I'UE). Le droit de l'Union économique et monétaire repose en effet sur la prémisse de l'autonomie budgétaire nationale (al. 218 sq.).

Le principe de démocratie joue un rôle central dans la politique d'intégration de l'Allemagne dans l'UE, régie par l'art. 23
de la Loi fondamentale. II s'agit en effet d'un des principes structurels que doit respecter l'évolution de l'UE afin que l'Allemagne puisse y contribuer et remplir ainsi l'obligation d'intégration faite par ce même article.

Si l'art. 23, § 1 est le pivot constitutionnel de “l'ouverture de l'Etat » allemand (offene Staatlichkeit) à son intégration dans l'UE, il n'en pose pas moins des conditions: "Pour l'édification d'une Europe unie, la République fédérale d'Allemagne concourt au développement de l'Union européenne qui est attachée aux principes fédératifs, sociaux, d'Etat de droit et de démocratie ainsi qu'au principe de subsidiarité et qui garantit une protection des droits fondamentaux substantiellement comparable à celle de la présente Loi fondamentale. A cet effet, la Fédération peut transférer des droits de souveraineté par une loi approuvée par le Bundesrat. L'article 79, al.2 et 3 est applicable à l'institution de I'Union européenne ainsi qu'aux modifications de ses bases conventionnelles et aux autres textes comparables qui modifient ou complètent la présente Loi fondamentale dans son contenu ou rendent possibles de tels compléments ou modifications ".

L'ouverture de la Loi fondamentale à I'intégration européenne découle également du préambule de la Loi fondamentale : "Conscient de sa responsabilité devant Dieu et devant les hommes, animé de la volonté de servir la paix du monde en qualité de membre égal en droits dans une Europe unie, le peuple allemand s'est donné la présente Loi fondamentale en vertu de son pouvoir constituant ».

En application de ces principes, la Cour, dans son arrêt de 2009 à propos du Traité de Lisbonne, avait défini le concept de la responsabilité d'intégration du Bundestag (voir REA 92/2009). A la suite de quoi a été adopté le 08-09-2009 la Loi sur la responsabilité d'intégration (Integrationsverantwortungsgesetz) précisant les modalités de l'action parlementaire.

En ce qui concerne l'articulation des pouvoirs entre l'échelon du Bundestag et celui de l'UE, il faut distinguer entre le droit primaire de l'UE (traités conclus par les Etats membres) et droit secondaire (actes des institutions européennes) :

- en ce qui concerne ledroit primaire, le Bundestag doit respecter la responsabilité d'intégration de la Loi fondamentale. Cela amène désormais le Tribunal constitutionnel fédéral à statuer, avant sa ratification par le parlement allemand, sur la conformité de tout nouveau traité avec les art. 38, 23 et 79, § 3 de la Loi fondamentale ;

- quant audroit secondaire, il relève par définition du domaine de compétences des organes de l'UE. Néanmoins, lorsqu'un acte législatif adopté par le Conseil de l'UE touche certaines compétences de l'Allemagne, cette disposition doit être soumise à l'approbation du Bundestag (réserve de constitutionnalité définie dans l'arrêt à propos du Traité de Lisbonne, voir REA 92/ 2009). En outre, le Bundestag, de même que les parlements des autres Etats membres, doit être consulté avant l'adoption de tout acte législatif, conformément au Protocole sur l'application du principe de subsidiarité et de proportionnalité du Traité de Lisbonne. S’il estime que le projet en question enfreint le principe de subsidiarité, il doit adresser aux présidents du Parlement européen, du Conseil et de la Commission un avis motivé (c'est par exemple ce que viennent de faire le Bundestag et le Bundesrat à propos du projet de la Commission d'instaurer un droit européen commun de la vente).

La Loi fondamentale garantit la vitalité du processus démocratique

Les juges ne peuvent pas se substituer au législateur
Autre considération digne d'être relevée : en formulant en termes prudents "qu'une modification démocratiquement légitimée de la configuration concrète des impératifs de stabilité que contient le droit européen (ne) serait (pas) a priori incompatible avec l'art. $79, \S 3$ de la Loi fondamentale ", la Cour fait allusion au fait que la Loi fondamentale est ouverte à des modifications ultérieures (al. 221). Et elle souligne expressément, en des termes remarquablement modernes qui ne sont pas sans rappeler le début de sa jurisprudence sur la constitutionnalité de l'ordre économique de la République fédérale ("arrêt sur l'aide à l'investissement " de 1954 ; voir Müller-Graff, 1984), que "l'art. 79, \& 3 de la Loi fondamentale... ne (garantit) pas le caractère immuable du droit en l'état, mais la pérennité de structures et procédures qui assurent la vitalité du processus démocratique et qui, ce faisant, garantissent également la responsabilité budgétaire globale du parlement » (al. 222).

En toute logique, la Cour en conclut donc qu'un engagement au respect de critères contraignants en matière de politique budgétaire, que celui-ci ait été pris à l'échelon national, européen ou international, n'est pas en soi ou a priori contraire à la démocratie, mais peut, bien au contraire, être nécessaire pour préserver dans la durée la latitude du jugement démocratique (al. 224, en référence à l'arrêt BVerfGE 129, pp. 124 et 170 ; et al. 225 sq. sur l'engagement européen et international). Et la Cour estime que cette question de la préservation ou de la limitation de la latitude d'action démocratique relève en priorité de la compétence et du jugement du législateur. L'al. 228 est ainsi rédigé : "Le Tribunal constitutionnel fédéral n’a pour mission de se substituer en la matière aux collectivités investies du pouvoir législatif dont c'est la compétence première » (en référence à l'arrêt BVerfGE 129, pp. 124 et 183). 
Autrement dit, le Tribunal constitutionnel fédéral, très soucieux de respecter la séparation des pouvoirs législatif et judiciaire, refuse avec beaucoup de sagesse de prendre position sur ce point en lieu et place du législateur démocratiquement élu auquel seul incombe la responsabilité du choix démocratique.

La Cour conforte donc l'autonomie de décision du législateur tout en soulignant par ailleurs que l'art. $79, \S 3$ de la Loi fondamentale stipule que toute modification de la Loi fondamentale " est interdite ». Or dans son arrêt à propos du Traité de Lisbonne, elle rappelait que, pour réaliser sa volonté, notamment dans le contexte de l'intégration européenne, le politique peut être amené à outrepasser ou enfreindre des principes dont la Cour considère l'intangibilité garantie par cet article. Toute progression dans l'intégration politique de l'UE amenant une modification de ces principes garantissant les domaines essentiels de la souveraineté exigerait donc que l'Allemagne se dote d'une nouvelle Constitution. L'art 146 de la Loi fondamentale stipule en effet: " $\mathrm{La}$ présente Loi fondamentale, qui, l'unité et la liberté de l'Allemagne ayant été parachevées, vaut pour le peuple allemand tout entier, devient caduque le jour de l'entrée en vigueur d'une Constitution adoptée par le peuple allemand en pleine liberté de décision ».
L'arrêt ne prend pas position sur l'avenir de la Constitution allemande

\footnotetext{
L'art. 79, § 3 de la Loi fondamentale stipule: "Toute modification de la présente Loi fondamentale qui toucherait à l'organisation de la Fédération en Länder, au principe du concours des Länder à la législation ou aux principes énoncés aux articles 1 et 20 , est interdite ".

Art. 1 de la Loi fondamentale :

(§ 1) "La dignité de l'être humain est intangible dignité de l'être humain est intangible. Tous les pouvoirs publics ont l'obligation de la respecter et de la protéger ».

(§2) "En conséquence, le peuple allemand reconnaît à l'être humain des droits inviolables et inaliénables comme fondement de toute communauté humaine, de la paix et de la justice dans le monde ».

(§3) "Les droits fondamentaux énoncés ci-après lient les pouvoirs législatif, exécutif et judiciaire à titre de droit directement applicable ».

L'art. 20 de la Loi fondamentale contient les principes au fondement de l'Etat, notamment :

(§ 1) "La République fédérale d'Allemagne est un État fédéral démocratique et social ".

(§ 2) “Tout pouvoir d'État émane du peuple. Le peuple l'exerce au moyen d'élections et de votations et par des organes spéciaux investis des pouvoirs législatif, exécutif et judiciaire ".

(§3) "Le pouvoir législatif est lié par l'ordre constitutionnel, les pouvoirs exécutif et judiciaire sont liés par la loi et le droit ».
}

Dans son arrêt à propos du Traité instituant le MES - contrairement à l'arrêt à propos du Traité de Lisbonne -, la Cour n'invoque pas explicitement l'art. 146 de la Loi fondamentale qui, de l'avis de la majorité des commentateurs, exigerait que la nouvelle Constitution soit adoptée par voie de referendum ; du reste, cette lecture est théorique, puisque la Loi fondamentale ne prévoit pas de recours à un referendum sur des questions de fond. La République fédérale d'Allemagne est une démocratie représentative.

\section{Irrecevabilité de certains des recours déposés.}

L'irrecevabilité d'un certain nombre des recours déposés découle des considérations exposées ci-dessus. II s'agit à l'évidence de l'affirmation selon laquelle l'approbation de l'art. 136, § 3 du Traité sur le fonctionnement de l'UE (explicitation du fait que l'institution d'un mécanisme - en l'occurrence le MES est compatible avec les dispositions relatives à l'UEM) violerait le droit de vote en le "vidant de sa substance". Malgré quelques imprécisions dans l'appréciation du droit primaire de I'UEM (al. 232-234), mais qui ne portent pas à conséquence dans l'affaire dont avait à connaître la Cour, l'arrêt reconnaît à juste titre que cette approbation ne mène pas à l'abandon de "l'objectif de stabilité intrinsèque de l'Union monétaire " (al. 233-234) et que la possibilité offerte de créer un mécanisme de stabilité n'aboutit pas à une perte de l'autonomie budgétaire nationale (al. 235-237).
Le MES n'entrave pas l'autonomie budgétaire nationale ni l'objectif de stabilité de I'UEM 
De même, après examen sommaire du référé en protection juridique, la Cour n'émet pas non plus de réserves sur la suspension du droit de vote des Etats membres de l'UEM en cas de refus de versement de leur part au mécanisme (cf. al. 262-266 sur les différents cas de figure prévus par le Traité instituant le MES). Elle estime qu'il est du pouvoir et du devoir du Bundestag de veiller en pleine autonomie à ce que les parts de l'Allemagne soient toujours versées intégralement et dans les délais (al. 268-270). Et en conséquence logique de la latitude d'appréciation du législateur, la Cour n'émet pas non plus de réserve constitutionnelle quant au montant de l'engagement de la République fédérale au sein du MES (al. 271) - à condition toutefois que soit respectée l'obligation constitutionnelle de fixer un plafond prévisible (voir infra).

La Cour ne formule pas non plus de réserve quant à la possibilité d'émettre ultérieurement des parts de capital supérieures à la valeur nominale : en droit constitutionnel, celle-ci n'entrave pas la responsabilité budgétaire globale (al. 272-279). Les dispositions nationales prises pour intégrer le Bundestag dans les processus de prise de décision relatifs au MES répondent pour l'essentiel aux exigences de la Loi fondamentale (al. 280-299). Il en va de même de la Loi sur la stabilité, la coordination et le pilotage au sein de l'UEM (al. 302-313) : cette loi d'approbation du Traité instituant le MES n'entrave en rien la responsabilité budgétaire globale du Bundestag (al. 314-317), et elle ne signifie pas non plus que la République fédérale serait contrainte de définir sa politique économique de manière irréversible (al. 318-319).

\section{II - Conformité à la Loi fondamentale du Traité instituant le MES}

La conformité du Traité avec la Loi fondamentale est au cœur de l'arrêt. Sur la base des exigences constitutionnelles développées dans son arrêt de 2011 sur les aides budgétaires dans la zone Euro (BVerfGE 129, pp. 124 et 185 sq. ; voir REA 102/2011), la Cour se livre à une minutieuse analyse pour parvenir à la conclusion logique que le Traité instituant le MES "ne répond à ces critères que si leur interprétation est conforme à la Loi fondamentale » (al. 241). Cette interprétation concerne la clarté de l'engagement financier global de la République fédérale et le droit d'information du Bundestag.

\section{La clarté du plafond des engagements (190 $024800000 €)$ découle du principe démocratique de l'auto-détermination et de la prévisibilité}

L'arrêt à propos du MES rappelle que les aides budgétaires décidées en 2010 ne pouvaient être considérées comme conformes à la Loi fondamentale qu'à une condition : à savoir "que l'engagement financier global de la République fédérale... était d'un montant ne dépassant pas certaines limites, ... que le Bundestag devait approuver isolément chaque mesure d'aide d'une certaine ampleur, que cette conditionnalité des aides lui revenait en propre et que ces aides étaient limitées dans le temps » (al. 241).

Garantie de prévisibilité

Le Tribunal constitutionnel fédéral applique dès lors ces principes au MES et, considérant l'art. 8, § 1 et 5 du traité qui l'institue, en conclut que le montant de $190024800000 €$ constitue " la limite supérieure de tous les engagements financiers résultant du Traité instituant le MES » et donc " la charge maximale susceptible de peser sur le budget fédéral » (al. 242). Si la Cour, respectueuse de la séparation des pouvoirs, ne se prononce pas sur la question de savoir si un engagement d'un tel montant est politiquement responsable, elle n'en reconnaît pas moins dans cette limite à ne pas dépasser le respect de la prévisibilité qu'exige le principe de l'auto-détermination démocratique.

Mais prudence quant à l'avenir...

Quant à la question de savoir si ce plafond peut être remis en question en application d'autres dispositions du Traité instituant le MES (émission de parts de capital d'une valeur supérieure à la valeur nominale en vertu de l'art. 8, § 2,4 
du Traité MES ; appel du capital autorisé en vertu de l'art. 9 ; possibilité d'un appel de fonds revu à la hausse selon l'art. 25, 2), elle est examinée de manière exhaustive et très précise (al. 245, 247-249 et 250 de l'arrêt). Si l'arrêt y répond par la négative dans un premier temps, il relativise toutefois cette lecture grâce à l'emploi de prudentes formulations du type : "pourrait valoir », " ne devrait pas s'opposer», " est susceptible de concerner", " devrait être exclu», "devrait se trouver limité », " ne devrait probablement pas se produire».

Enfin, le Tribunal constitutionnel fédéral se penche précautionneusement sur de possibles « interprétations susceptibles de faire l'objet de dubitation » (al. 251253) et dévoile sans pitié le flou terminologique et l'imprécision systématique du Traité instituant le MES. Cet état de fait pourrait s'expliquer à notre avis par l'emploi délibéré d'un langage abstrait pour en dissimuler la portée ou tout simplement par une mauvaise habitude très répandue et qui veut que ce genre de texte soit dans un premier temps formulé en anglais, une langue qui manque cruellement de précision juridique. Quoi qu'il en soit, la Cour émet des considérations systématiques et téléologiques et parvient à la conclusion qu'est également envisageable une interprétation du Traité «qui ne serait pas compatible avec l'exigence constitutionnelle selon laquelle les engagements budgétaires doivent être fixés en toute clarté et définitivement arrêtés »(al. 251).

\section{Le droit d'information du Bundestag découle de la responsabilité d'intégration du parlement}

Dans la continuité de sa jurisprudence, le Tribunal constitutionnel fédéral analyse également le Traité instituant le MES pour savoir s'il respecte le droit d'information du Bundestag exigé par la Loi fondamentale. Une analyse en profondeur des dispositions afférentes que sont l'inviolabilité des documents et archives du MES (art. 32, §5 du Traité), le secret professionnel auquel sont tenus les membres de ses conseils et ses collaborateurs (art. 34) et leur immunité (art. 35, § 1), amène la Cour à la conclusion que «les parlements des Etats membres, ... en tant qu'ils portent la souveraineté budgétaire et que, visà-vis de leurs citoyens, ils ont à porter jusque dans leur exécution la responsabilité des engagements pris dans le cadre du MES... ne font pas partie des tiers devant être exclus du flux d'information » (al. 257 de l'arrêt). Par cette interprétation conforme à la Loi fondamentale, le Tribunal prend position contre la création d'une institution internationale d'aide budgétaire fonctionnant en cercle fermé et échappant à tout contrôle parlementaire. II reconnaît toutefois que le MES et d'autres Etats qui en sont membres (al. 258) puissent se livrer à une interprétation différente, qui ne serait alors pas conforme à la Loi fondamentale.

Dans le cas précis de l'affaire dont avait à connaître la Cour, le droit d'information du Bundestag découle du principe constitutionnel de la responsabilité budgétaire globale du parlement (un des domaines essentiels de souveraineté définis dans l'arrêt à propos du Traité de Lisbonne). L'idée sousjacente est simple, et bien connue dans le droit des sociétés : une personne habilitée à prendre des décisions ne peut exercer pleinement cette responsabilité que si elle est suffisamment informée. Le présent arrêt applique ce principe au MES : le Bundestag doit donc être suffisamment informé ; la Loi fondamentale interdit donc que le secret professionnel s'applique à lui. La conséquence de ce raisonnement au cœur de cet arrêt est que la Loi fondamentale exige que ce droit soit garanti en droit international, faute de quoi la République fédérale ne peut être liée par le Traité instituant le MES.

\section{III - Obligation faite aux organes politiques allemands de prendre des garanties en droit international}

... étant donné le flou du Traité
L'information est le préalable de l'exercice de la responsabilité 


\section{contre tout engagement de l'Allemagne découlant d'une interprétation divergente du traité MES}

Rappelons que le contrôle en constitutionnalité du Traité instituant le MES n'est, au fond, qu'une procédure interne, c'est-à-dire sans effets contraignants en externe : il ne lie pas les autres Etats signataires. Déjà, le Traité de Lisbonne avait donné lieu à un tel contrôle en constitutionnalité (arrêt rendu le 30 juin 2009 ; voir REA 92/2009). Les dispositions alors interprétées à la lumière de la Loi fondamentale concernaient le périmètre à accorder à certaines nouvelles normes en matière de compétences de I'UE (BVerfGE 123, p. 267 ; plus particulièrement pp. 358-399).

L'arrêt « Lisbonne » définissait des réserves, l'arrêt MES exige de les transcrire en droit international

Nouveauté : une méthode préventive...

... pour garantir le droit d'information du législateur Adoption d'un
protocole annexe au Traité
Mais lorsqu'il cherche à asseoir son interprétation de la conformité avec la Loi fondamentale des engagements pris par l'Allemagne dans le domaine du droit international, l'arrêt rendu à propos du Traité MES se meut en terrain inconnu en comparaison avec celui rendu à propos du Traité de Lisbonne. Dans ce dernier, le Tribunal constitutionnel fédéral s'était contenté, en renvoyant à l'exigence de contrôle a posteriori auquel il se considère seul habilité à procéder, de déclarer non contraignantes pour la République fédérale certaines mesures de l'Union européenne dès lors que cette dernière les avait prises en outrepassant ses compétences (constat " ultra vires »; BVerfGE 123, 267, p. 240 et p. 339). La conséquence en était que nulle mesure ultra-vires prise ultérieurement ne pourrait s'appliquer en Allemagne (voir REA 92/2009). Mais la Cour ne faisait pas encore injonction à la République fédérale de transcrire ces réserves en droit international. C'est là la nouveauté qu'apporte l'arrêt à propos du MES : il fait obligation aux organes politiques de la République fédérale de garantir ( $\mathrm{pp} .251,253,254$ et 259) que les matières définies cidessous et contenues dans le Traité MES trouvent en droit international une interprétation conforme à la Loi fondamentale, et ce à titre préventif. Ces matières sont la limite supérieure de l'engagement pris par la République fédérale dans le cadre du Traité instituant le MES et le droit d'information du Bundestag.

La méthode choisie dans cet arrêt pour asseoir le contrôle en constitutionnalité diffère foncièrement de celle utilisée dans l'arrêt à propos du Traité de Lisbonne : elle n'est pas répressive, mais au contraire préventive. C'est ainsi que, avec de nombreuses variantes dans la formulation, le Tribunal constitutionnel fédéral déclare qu'il incombe à la République fédérale le devoir "d'écarter dans le cadre de la procédure de ratification en droit international » tout doute quant à l'interprétation à accorder à la limite supérieure de son engagement (al. 251) ou - e c'est là une décision sans appel - à « faire valoir clairement qu'elle ne peut se considérer liée à la totalité du Traité instituant le MES au cas où les réserves qu'elle fait valoir se révéleraient invalides " (al. 253).

En ce qui concerne la garantie de l'information du Bundestag, requise pour le contrôle du MES, l'arrêt exige " ici aussi de garantir en droit international une interprétation compatible avec la Loi fondamentale » (al. 254). Et il déclare qu'une ratification par l'Allemagne du Traité instituant le MES n'est autorisée que si la République fédérale " assure une interprétation du traité garantissant que le Bundestag et le Bundesrat disposent des informations indispensables pour forger leur opinion et prendre leurs décisions»(al. 259) ou si elle «fait valoir avec clarté qu'elle ne peut se considérer aucunement liée par le Traité instituant le MES si la réserve qu'elle formule se révèle invalide » (al. 259).

Ces exigences n'obligent pas l'Allemagne à recourir à un moyen particulier dans le domaine du droit international. Une déclaration unilatérale de réserve ne constituerait toutefois pas une garantie suffisante pour une interprétation du Traité conforme à la Loi fondamentale (voir F.A.Z., 20-09-2012). II est permis par ailleurs de douter que les autres signataires du Traité puissent procéder à une interprétation conforme à la Loi fondamentale allemande (ibid.). Seul un 
protocole annexe soumis à la ratification de tous les Etats membres du MES serait donc en mesure d'apporter la sécurité requise. Mais d'un autre côté, un Etat devrait être délié de ses engagements s'il déclare sans ambiguïté qu'il ne désire pas être partie contractante si sa réserve n'est pas valide (ibid.). Dans ce cas, toute revendication ou tout comportement divergent de la part du MES ou d'un de ses Etats membres serait alors contraire aux règles de loyauté.

\section{IV - Report de la question de la non conformité au droit de la politique d'aide budgétaire adoptée par la BCE}

En ce qui concerne l'évolution à venir de la politique d'aide budgétaire internationale, il convient de relever le report, en attendant le jugement de fond (al. 202), de la plainte en non conformité de la politique d'aide budgétaire adoptée par la Banque centrale européenne ("actes juridiques débordants »).

Néanmoins, l'arrêt rendu en référé à propos du MES souligne avec une surprenante récurrence et une étonnante clarté l'interdiction de la monétarisation de la dette des Etats par la BCE inscrite à l'art. 123 du Traité sur le fonctionnement de l'UE (al. 219, 220, 233 et 276-278). S'y ajoute l'observation expresse selon laquelle " est également interdit le contournement de l'interdiction d'une monétarisation de la dette ... par l'acquisition d'obligations d'Etat sur le marché secondaire par la (BCE) et visant un financement budgétaire des Etats membres en indépendance des marchés de capitaux » (al. 278 en référence au septième considérant du Règlement (CE) $n^{\circ} 3603 / 93$ du Conseil du 13-12-1993, Journal officiel $n^{\circ} L 332$ du 31/12/1993 $p$. 1).

\section{La problématique}

Sous l'angle du droit primaire de l'UE, la problématique que représente la politique d'acquisition d'obligations d'Etat annoncée par la BCE est thématisée depuis 2010 (voir Müller-Graff, 2010). L'art. 123, §1 du Traité sur le fonctionnement de l'UE interdit à la BCE « l'acquisition directe des instruments de la dette » notamment des Etats membres de la zone Euro, c'est-à-dire leur achat sur le marché primaire. Autrement dit, cet article interdit de lancer la " planche à billets " pour soutenir les budgets nationaux à l'instar de ce que pratiquent certaines banques centrales hors de I'UE. Cette disposition a pour objectif d'aider les Etats à tenir leurs engagements en matière de "prévention des déficits publics excessifs " (art. 126, § 1 du Traité sur le fonctionnement de I'UE). Elle a aussi pour but de réduire le risque de pertes pour les détenteurs du capital de la BCE ; en effet, l'art. 28.2 du Protocole sur les statuts du Système européen de banques centrales et de la BCE stipule : "Les banques centrales nationales sont seules autorisées à souscrire et à détenir le capital de la BCE ".

Il découle à l'évidence de la manière dont sont formulées ces dispositions le droit, pour la BCE, d'acquérir « indirectement » des créances, c'est-à-dire sur le marché secondaire, voire le droit de racheter des obligations d'Etat dans le portefeuille d'investisseurs primaires, notamment des banques et des hedgefonds. Le sens véritable de cette autorisation de rachat sur le marché secondaire ne peut résider que dans la présupposition que les investisseurs du marché primaire ont procédé, avant l'acquisition d'obligations d'Etat, à une évaluation des risques dans les règles de l'art, c'est-à-dire en commerçants avisés.

Or quand, comme l'a annoncé son président le 6 septembre 2012, la BCE envisage de lancer une politique d'acquisition d'obligations émises par les Etats aux prises avec la crise de leur dette, ce mécanisme de sécurité qu'est l'évaluation indépendante des risques par les acteurs du marché primaire se trouve désactivé. De ce fait, la conjugaison entre l'annonce préalable (quelle
Rappel de l'interdiction de monétariser la dette

Il est interdit à la BCE d'acheter directement des obligations d'Etat, ...

... mais pas indirectement

L'inconnue juridique de la nouvelle politique de la BCE 
Karlsruhe, première instance

Renvoi devant la CJUE ? qu'en soit la forme concrète) d'un rachat sur le marché secondaire puis sa réalisation doit être considérée comme une tentative de contourner l'interdiction d'acquérir des obligations d'Etat sur le marché primaire. Reste à clarifier les conséquences juridiques d'une telle appréciation de la politique de la BCE. De deux choses l'une : s'il s'agit de la mise à profit d'une simple « lacune » dans le droit primaire, celle-ci n'entraîne aucune conséquence juridique ; si ce n'est pas le cas, alors l'acte d'achat est frappé de nullité.

\section{Le rôle du Tribunal constitutionnel fédéral}

Le Tribunal constitutionnel fédéral peut être amené à jouer un rôle clé dans la clarification de la question de savoir si la nouvelle politique de la BCE constitue une violation du droit primaire de l'UE ou non. En effet, tant que la Cour européenne de justice n'est pas saisie d'un recours en nullité afférent en vertu de l'art. 263 du Traité sur le fonctionnement de l'UE, c'est au Tribunal constitutionnel fédéral qu'il revient, en première instance, de statuer en la matière, du moins si cette question est d'importance pour le jugement de fond attendu (cela n'est pas certain).

Si la Cour de Karlsruhe parvient à la conclusion que les mesures prises par la BCE contreviennent à l'art. 123, § 1 du Traité sur le fonctionnement de l'UE et que la BCE outrepasse ce faisant ses compétences ("ultra vires ») et ce, dans une mesure d'importance structurelle (au sens de la décision « Honeywell » du 6 juillet 2010), alors, en vertu de l'art. 267, § 3 du Traité sur le fonctionnement de I'UE, elle serait tenue de renvoyer l'affaire devant la Cour européenne de justice. Avec la décision « Honeywell », le Tribunal constitutionnel fédéral a prouvé qu'il était prêt à le faire (BVerfG, décision du 6 juillet 2010 (BvR 2661/06) = BVerfGE 126, p. 186).

En 2010, le Tribunal constitutionnel fédéral avait rendu cette décision « Honeywell » en réponse aux vives critiques suscitées par son arrêt à propos du Traité de Lisbonne. Le choix de formulations parfois très arrêtées risquait en effet de donner l'impression que la Cour pourrait être tentée de s'arroger le droit d'interpréter selon sa jurisprudence les normes de compétence du droit primaire de l'UE et, à chaque fois qu'elle constaterait un débordement dans une disposition européenne, déclarer celle-ci non contraignante pour l'Allemagne.

La décision « Honeywell » relativise cette lecture :

- le Tribunal constitutionnel fédéral déclare que, à chaque fois qu'il parviendra à un constat ultravires, il soumettrait immédiatement l'affaire à la Cour européenne de Justice ;

- il explique en outre qu'il ne considérerait pas comme non applicable ou non contraignante toute mesure de l'UE outrepassant ses compétences, mais qu'il limiterait ce verdict aux cas graves, c'est-à-dire aux «glissements de compétences d'ampleur structurelle » - un concept, à l'évidence, qui laisse à la Cour une grande marge d'appréciation.

Au vu de la manière de procéder de la BCE comme de la dimension de sa nouvelle politique d'acquisition, il n'est donc nullement à exclure que cette dernière soit l'objet du premier renvoi opéré par la Cour de Karlsruhe auprès de la Cour européenne de justice. Et c'est alors à la Cour de Luxembourg qu'il reviendra de se préoccuper de la politique d'aide budgétaire pratiquée par la BCE. Videant consules ne quid detrimenti respublica capiat! (Que les consuls veillent à ce que la République ne subisse aucun dommage, selon l'adage des Romains)...

DANS CET ARRÊT D'UNE GRANDE SAgesse, le Tribunal constitutionnel fédéral se montre fidèle à sa ligne de défense du principe de démocratie. II assure et concrétise ce principe national jusque dans les évolutions les plus récentes de l'intégration européenne, en l'occurrence l'adoption du Traité instituant le MES, mais sans pour autant contrecarrer foncièrement les nouvelles orientations européennes du législateur allemand démocratiquement légitimé.

Depuis son arrêt rendu à propos du Traité de Maastricht (1993, BVerfGE 89), le Tribunal constitutionnel fédéral a renforcé le principe de démocratie dans le 
contexte de l'intégration européenne en considérant le droit de vote individuel aux élections du Bundestag (art. 38 de la Loi fondamentale) comme un droit fondamental autorisant tout électeur de saisir le Tribunal constitutionnel fédéral s'il estime qu'il est lésé par un traité international. L'idée sous-jacente est celleci : la composante matérielle du droit de vote se trouve vidée de son contenu si le Bundestag se voit retirer la substance de son pouvoir de décision par le transfert de compétences à une organisation internationale. Jusqu'à présent, la Cour n'a jamais émis de réserves quant à la conformité avec la Loi fondamentale des transferts opérés à l'échelon de l'UE. Néanmoins, pour la première fois, elle a insisté, dans son arrêt à propos du Traité de Lisbonne, sur une interprétation conforme de certaines compétences transférées: coopération des juridictions en droit civil et pénal, droit communautaire du commerce, politique extérieure et politique de sécurité communes.

Tout en s'inscrivant dans la continuité des grands principes constitutionnels au fondement de sa jurisprudence à propos des récentes avancées en matière d'intégration européenne (notamment les arrêts à propos du Traité de Lisbonne et des aides budgétaires au sein de la zone Euro), le Tribunal constitutionnel fédéral apporte une nouvelle respiration et une nouvelle souplesse au processus d'intégration européenne. Son analyse du Traité instituant le MES est remarquable et louable. Juridiquement très fouillée, elle est d'une grande clarté : elle renonce à toute rhétorique abstraite et juridiquement inutile à propos de la souveraineté nationale comme à l'évaluation de problématiques hypothétiques et abstraites.

Ainsi, l'arrêt ouvre intelligemment une nouvelle voie pour résoudre à l'avenir tout conflit opposant le principe démocratique allemand et les avancées à venir en matière de politique européenne. Surtout, il prouve que le respect de la séparation des pouvoirs au sein d'un Etat (notamment al. 223, 234 et 271) ouvre des perspectives à l'exercice de la raison pratique d'un contrôle judiciaire sur les mesures politiques. Les seules faiblesses de cet arrêt concernent sa perception du droit de l'UEM, notamment celle de l'art. 125 du Traité sur le fonctionnement de l'UE et la clause du "no bail-out » dont l'arrêt ignore en partie les exégèses (al. 232-236) ; mais ces faiblesses sont au fond sans conséquence pour le jugement et son argumentation.

Enfin, en ce qui concerne l'éternelle question de savoir quel est l'instrument le plus approprié pour le contrôle a posteriori des décisions prises par les organes représentatifs en démocratie, cet arrêt illustre le potentiel, mais aussi les limites, d'un contrôle juridictionnel en comparaison d'un referendum dans une démocratie représentative.

(Traduction : I. Bourgeois)

\section{Indications bibliographiques}

\footnotetext{
- Bourgeois I., «La constitutionnalisation de l'ordre économique. Les 50 ans du Bundesverfassungsgericht », Regards sur l'économie allemande, n`92, juillet 2009

- «L'Europe à l'épreuve des tribunaux », interview d'Andreas Vosskuhle, président du Tribunal constitutionnel fédéral, Le Monde, 02-10-2012

- MAdeLIN TH., "Crise en Europe : la Cour de Karlsruhe sous une pression historique », Les Echos, 12-09-2012

-MüLleR-GRAFf P.-C., « 60 Jahre Grundgesetz aus der Sicht des Europarechts », in Jahrbuch des öfentlichen Rechts, Neue Folge vol. 59 (2011)

- MüLleR-Graff P.-C., "L'arrêt de Karlsruhe sur les aides budgétaires dans la zone Euro ", Regards sur l'économie allemande, $n^{\circ} 102 / 2011$

-MüLleR-Graff P.-C., « Einander ausgeliefert », Frankfurter Allgemeine Zeitung, 20-05-2010

-MüLleR-Graff P.-C., "L'arrêt de Karlsruhe sur le Traité de Lisbonne », Regards sur l'économie allemande, $\mathrm{n}^{\circ} 92 / 2009$

-MüLleR-GRAFF P.-C., Unternehmensinvestitionen und Investitionssteuerung im Marktrecht, Tübingen, 1984
} 
-TALMON ST., « Unter Vorbehalt », Frankfurter Allgemeine Zeitung, 20-09-2012 - www.bundesverfassungsgericht.de

Arrêt du 12-09-2012:

http://www.bundesverfassungsgericht.de/entscheidungen/rs20120912 2bvr139012.html

Communiqué de presse :

http://www.bundesverfassungsgericht.de/pressemitteilungen/bvg12-067.html; 



\section{Allemagne, les chemins de l'Unité.}

\section{Vient de paraître aux Editions du CIRAC} en douze tableaux

\section{Reconstruction d'une identité}

\author{
Sous la direction \\ d'Isabelle Bourgeois \\ Travaux et documents du CIRAC \\ Juillet 2011 \\ ISBN 978-2-905518-41-5 \\ 276 p., $25 €$
}

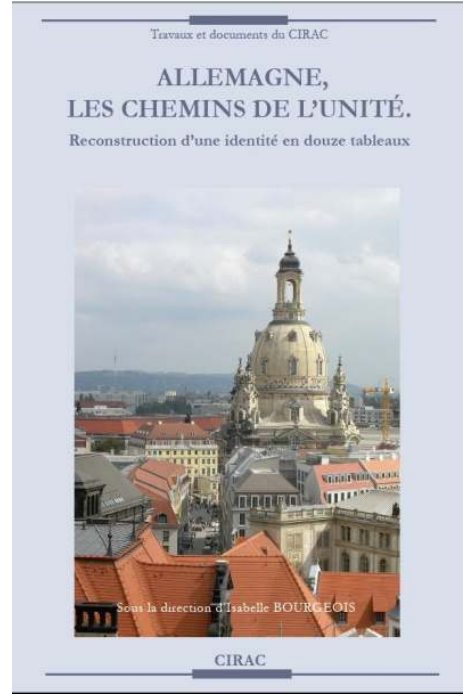

Depuis quelque temps, notre partenaire privilégié dans l'Union européenne nous fascine et nous irrite à la fois. Première puissance économique européenne, l'Allemagne semble aujourd'hui abandonner cette retenue qui caractérisait jusqu'ici sa politique extérieure et européenne pour exercer un leadership politique de fait dans une Europe qui se cherche.

Il est vrai qu'en cela l'Allemagne défend désormais, plus que par le passé, ses intérêts nationaux légitimes. Cette évolution ne traduit pas tant la volonté d'affirmer une quelconque suprématie politique puisque la République fédérale, qui n’a reconquis sa pleine souveraineté qu'en 1990 avec l'Unité allemande, en a alors immédiatement rétrocédé une grande partie à l'UE dans des domaines vitaux, au premier rang desquels celui de la monnaie. Le fait qu'elle tienne désormais à jouer pleinement son rôle dans le gouvernement partagé de l'Union procède plutôt d'une nouvelle identité qui a émergé au fil des générations.

Cette identité se nourrit de l'expérience des épreuves surmontées, et surtout de la confiance acquise dans la capacité des décideurs publics et privés, comme de la société dans son ensemble, à édifier un système démocratique, économique, social et monétaire durable qui résiste aux chocs d’un monde imprévisible et instable. Assumant pleinement leur passé, les Allemands ont en même temps acquis la conscience du chemin parcouru, de la stabilité de ce qu'ils ont reconstruit, et aussi de la nécessité de le préserver en affrontant courageusement l'avenir. C'est cette identité collective qui explique que l'Allemagne se présente, aujourd'hui, pleinement sowveraine en elle-même, c'est-à-dire consciente de ses acquis et de son expérience, fière de ses principes et de ses valeurs, confiante dans sa capacité à proposer des choix aux Européens et à en assumer les responsabilités.

Comment cette nouvelle Allemagne «souveraine» s'est elle construite, et sur quels fondements ? C'est ce cheminement, à trajectoires multiples et imbriquées, que tentent de retracer et d'éclairer les contributions de cet ouvrage. En douze tableaux, les auteurs, spécialistes éminents ou experts confirmés associés au « réseau de compétences » du CIRAC, présentent la (re)construction patiente et raisonnée des fondations, le séisme libérateur et créateur que fut la réunification allemande et européenne, et les perplexités d'un pilotage à plusieurs dans un monde tout à la fois global et multipolaire.

A la lecture de ces tableaux, il apparaît surtout que ce qui fait l'unité de l'Allemagne d'aujourd'hui, et en même temps sa réussite, c'est en réalité une dynamique: celle de la quête permanente d'un équilibre durable dans l'acceptation et la gestion collectivement assumées du changement. Un bilan éclairant pour une Europe perplexe.

AVANT-PROPOS

\section{SOMMAIRE}

INTRODUCTION

Alfred Grosser : II était une fois un petit Etat provisoire...

\section{I- Prospérité et STABILITÉ}

Hans-Helmut Kotz : 60 ans de Loi fondamentale, 61 ans de Deutsche Mark

Henrik Uterwedde : L'économie sociale de marché : la jeunesse d'un référentiel

Siegfried Schieder : La politique européenne de la RFA en quête de nouveaux équilibres

II - L'UNITÉ : ÉLARGISSEMENT ET APPROFONDISSEMENT

Joachim Schild : 20 ans après l'Unité : la France et l'Allemagne dans la nouvelle Europe

Karl Brenke : 20 ans après l'Unité : l'Allemagne de l'est reste économiquement à la traîne

Uwe Kammann : Compagnons de voyage. Les médias et l'Unité allemande

Isabelle Bourgeois : Les médias dans l'Allemagne unie. De l'unification démocratique à la normalisation du marché Thomas Petersen : Frères et sœurs dissemblables. UN bilan de l'Unité allemande

III - Une NOUVElle Allemagne?

Eugen Spitznagel : Un marché de l'emploi en mutation

René Lasserre : La réforme de l'Etat social en Allemagne

Hans Stark : Les métamorphoses de la politique étrangère allemande 1949-2011

Thomas Petersen : Une nouvelle identité allemande? 